approach, however, should be to collect epithelial cells without excess vesicle fluid, because this may contain viral antigens that react with the monoclonal antibodies and produce a green haze over the slide, making it difficult to interpret. On the other hand, once the lesion is ulcerated, infected cells are less numerous, and numbers sufficient to provide an adequate sample difficult to obtain, their collection proving painful for the patient. Despite these problems, however, the value of the direct test seems obvious. Apart from its ability to give a result within 30 minutes of the specimen being taken, it was found to be sensitive if an adequate specimen was obtained; and there was only one occasion on which the direct test was positive but HSV was not isolated in culture. HSV was isolated, however, from this patient on a second sampling. The typing of HSV by the direct test was also specific. We do not forsee this technique replacing cell culture procedures, but it seems logical to use the two in combination; a sample taken for examination in cell culture at the same time as one for the direct test could be discarded if the latter specimen was adequate, which our results indicate would occur in about $40 \%$ of cases.
References

I Reeves WC, Corey L, Adams HG, Vontver LA, Holmes KK. Risk of recurrence after first episodes of genital herpes. Relation to HSV type and antibody respense. $N$ Engl J Med 1981;305:315-9.

2 Crumpacker CS, Schnipper LE, Zaia JA, Levin MJ. Growth inhibition by acycloguanosine of herpesvirusess isolated from human infections. Antimicrob Agents Chemother 1979;15: 642-5.

3 De Clercq E, Descamps J, Verhelst G, et al. Comparative efficacy of antiherpes drugs against different strains of herpes simplex virus. J Infect Dis 1980;141:563-74.

4 Brown ST, Jaffe HW, Zaidi A, et al. Sensitivity and specificity of diagnostic tests for genital infection with herpes virus hominis. Sex Transm Dis 1979;6:10-3.

5 Moseley RC, Corey L, Benjamin D, Winter C, Remington ML. Comparison of viral isolation, direct immunofluorescence, and indirect immunoperoxidase techniques for detection of genital herpes simplex virus infection. J Clin Microbiol 1981;13:913-8.

Requests for reprints to: Dr D Taylor-Robinson, Division of Sexually Transmitted Diseases, Clinical Research Centre, Watford Road, Harrow, Middlesex HAl 3UJ, England.

\section{Rapid immunofluorescence staining of human renal biopsy specimens using microwave irradiation}

$\mathrm{K}$ Y CHIU, $\mathrm{K}$ W CHAN From the Histopathology Unit, Department of Pathology, University of. Hong Kong, Queen Mary Hospital Compound, Hong Kong

Immunological studies of renal tissue are indispensable both in clinical practice and in experimental glomerulonephritis. Immunofluorescence is the usual technique used to measure concentrations of immunoglobulins and complements when diagnosing renal disease, especially IgA nephropathy and membranous glomerulonephritis. ${ }^{1-4}$ Immunofluorescence is performed on fresh frozen sections, ${ }^{5}$ and detachment of the section from the glass slide is a common problem during incubation and washing, especially when the sections have been incompletely dried. To ensure firm adherence the cryocut sections are usually dried for about 30 minutes at room temperature and then incubated with the fluorescein conjugated antibodies for another 30 minutes. $^{5}$ We describe a method in which microwave irradiation is used for the

Accepted for publication 19 November 1986 rapid drying of frozen sections and incubation of fluorescein conjugated antisera.

\section{Material and methods}

Human renal tissue obtained by percutaneous needle biopsy was divided into three portions for light microscopy, electron microscopy, and immunofluorescence staining. For immunofluorescence, fresh renal tissue was embedded in ornithine carbonyl transferase medium and snap frozen in liquid nitrogen. Cryostat sections were cut at $6 \mu \mathrm{m}$, and spare sections for this study were stored at $-30^{\circ} \mathrm{C}$ before immunostaining. We selected 12 cases for study after the definitive diagnoses had been made and the formal pathology reports had been issued; six showed IgA nephropathy, four active diffuse proliferative lupus nephritis, and two showed idiopathic membranous glomerulonephritis. Before immunostaining, the frozen sections were placed horizontally on the rim of the carousel-turntable in a domestic microwave oven.(Sharp Model R-7.200, Japan) and irradiated for $(12,36,48)$ seconds and (one, two, five, 10, 20) minutes at the medium to low (defrost) setting for drying. The oven operated at $2450 \mathrm{MHz}$ and had a maximum output of $600 \mathrm{~W}$. After microwave defrosting the sections were washed in two changes of phosphate buffered saline (PBS) $\mathrm{pH} \mathrm{7.4}$ for two 
minutes each. They were then incubated with $60 \mu \mathrm{l}$ fluorescein isothiocyanate (FITC)-labelled rabbit antihuman IgA, IgG, IgM, C3 and Clq (Behringwerke AG, Germany), diluted $1 / 5$ in PBS and irradiated again for between 12 seconds and two minutes at the medium to high setting in the oven. After three further washes in PBS of three minutes each, the sections were mounted in buffered glycerol and examined under a Nikon epifluorescence microscope.

As controls, two sets of frozen sections were dried completely at room temperature for 30 minutes and subjected to immunofluorescence staining under the conditions described before, but at room temperature and without microwave irradiation, and conventional immunofluorescence staining for comparison. In a second control group PBS was substituted for FITCconjugated antisera.

\section{Results}

Frozen sections were adequately dried after microwave irradiation for one minute at the medium to low setting. Shorter exposure resulted in incomplete drying of sections, which carried a risk of detachment of sections from slides during subsequent incubation. The table shows the effect on immunofluorescent staining of various exposure times to microwave irradiation at the medium to high setting after microwave defrosting. The optimal exposure consistent with maximum staining intensity and minimum background staining was 48 seconds.

All cases of IgA nephropathy showed predominant coarsely granular to lumpy mesangial IgA deposition in the glomeruli (fig la). The staining intensity and resolution were comparable with those produced by the conventional procedure with similar antibody dilution. The pattern and location of immunoglobulin deposition after microwave irradiation were identical with those seen in sections treated conventionally in all 12 cases. Longer exposure resulted in increased background staining and poor morphology, due to drying of antisera during microwave incubation. In addition to strong staining intensity, microwave irradiated sections showed firm, uniform adhesion to glass slides without any adhesives, as well as good preservation of tissue morphology. Incubation of FITC-conjugated antisera for 48 seconds at room temperature produced negative staining in the controls (fig lb).

\section{Discussion}

The use of microwave irradiation for tissue fixation and histological staining for light and electron microscopic studies is increasing. ${ }^{6-11}$ It has also been used to improve immunoperoxidase staining of lymphocyte antigens. ${ }^{12}$ Microwave energy is a non-ionising electromagnetic wave that increases the molecular movement of dipolar molecules such as water. It provides a means of generating heat rapidly, and facilitates the formation of antigen-antibody complexes. The use of microwave irradiation in immunofluorescence therefore, not only produces rapid drying and firm adherence of the cryocut sections to the slides, but also shortens the time needed for immunostaining. Current methods recommend either drying

Table Effect of exposure time to microwave irradiation on immunofluorescence staining

\begin{tabular}{|c|c|c|c|c|c|c|}
\hline \multirow[b]{3}{*}{ Exposure time (seconds) } & \multicolumn{5}{|c|}{ Staining intensity of } & \multirow[b]{3}{*}{ Background } \\
\hline & \multicolumn{3}{|c|}{ Immunoglobulins } & \multicolumn{2}{|c|}{ Complements } & \\
\hline & $A$ & $G$ & $M$ & $\mathrm{Clq}$ & $C 3$ & \\
\hline 0 & - & - & - & - & - & - \\
\hline 12 & + & + & $t$ & + & + & - \\
\hline 24 & ++ & ++ & + & ++ & + & - \\
\hline 36 & ++ & ++ & ++ & ++ & ++ & - \\
\hline 48 & +++ & +++ & +++ & +++ & +++ & - \\
\hline 60 & +++ & +++ & +++ & +++ & +++ & + \\
\hline 90 & ++ & D & D & ++ & D & ++ \\
\hline 120 & D & D & D & D & D & ++ \\
\hline
\end{tabular}

Staining intensity: - negative; + weak positive; ++ moderate positive +++ strong positive. $D$ : applied antisera dried during incubation. 

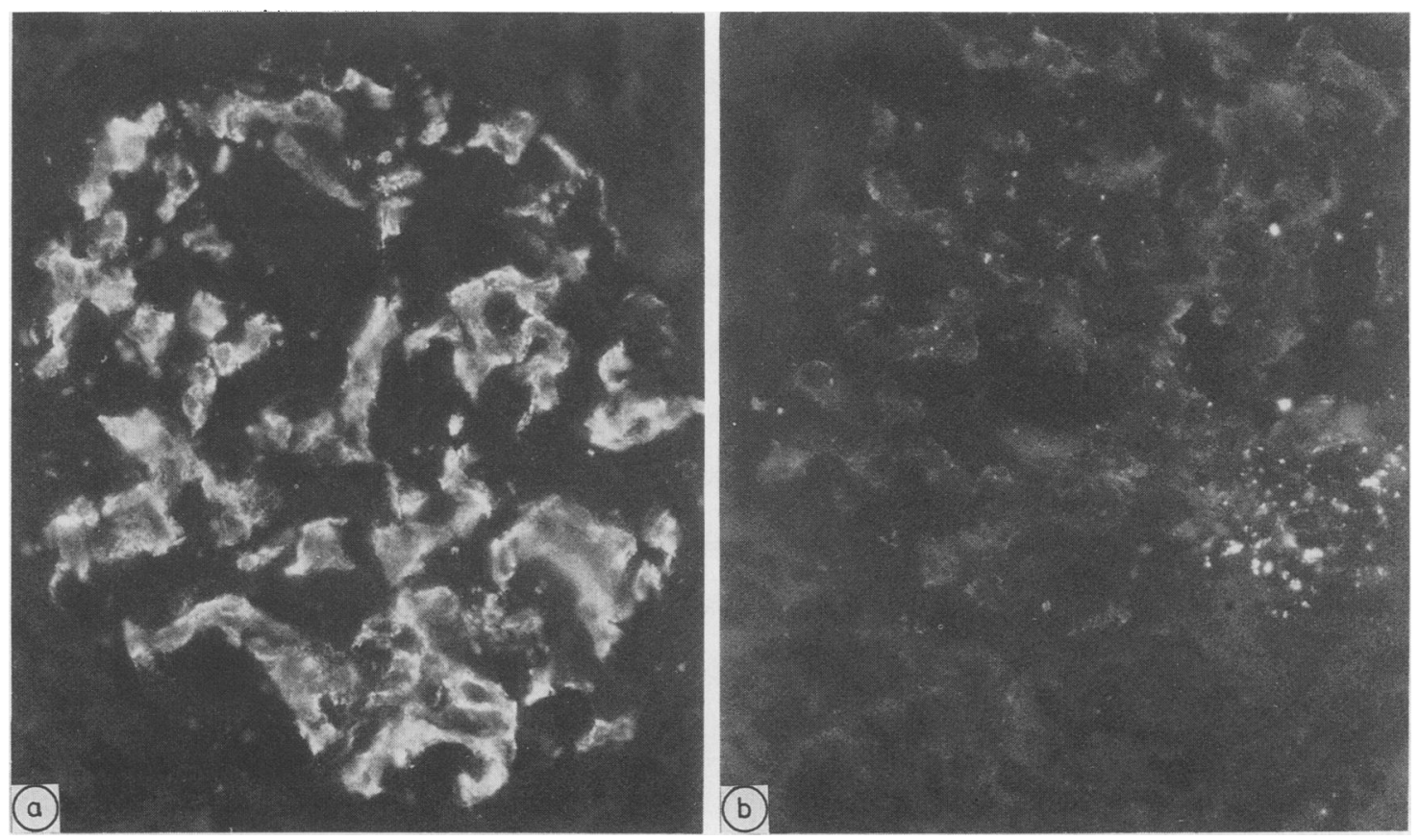

Fig 1 (a) Microwave irradiated immunofluorescence staining of frozen section of renal biopsy for IgA;

(b) Immunofluorescence staining of glomerulus for IgA at room temperature and without microwave irradiation.

of frozen sections at room temperature for 30 minutes or at $37^{\circ} \mathrm{C}$ for 15 minutes; the time is reduced to one minute if the microwave oven is used. Although the sections can tolerate irradiation of up to five minutes at the medium to low setting during the drying stage, considerable loss of protein antigenicity occurs if this time is exceeded. The reason for using medium to low, and not higher temperature settings, is to permit gradual defrosting and the escape of water molecules without steam formation, which distorts tissue morphology. The incubation period for the FITCconjugated antibody, which normally takes 30 minutes at room temperature, is completed in $48 \mathrm{sec}-$ onds in the microwave oven at the medium to high setting. Care must be taken not to overheat the sections; a temperature higher than medium to high, or an incubation time longer than one minute, would cause rapid boiling and drying of the applied antisera, resulting in increased background staining, poor tissue morphology, and the detachment of sections from slides. To ensure maximum and even irradiation of sections all slides should be placed horizontally on the rim of the turntable.

Due to the lack of suitable biopsy material, this method has only been tested in cases of immune complex nephritis. It is now necessary to test it in cases of antiglomerular basement membrane nephritis to show that it can maintain a linear pattern of immunoglobulin deposition. The typical peripheral granular pattern of immunoglobulin deposition in membranous glomerulonephritis has been successfully shown after microwave irradiation in the two cases studied.

In conclusion, the use of microwave irradiation in immunofluorescence staining of renal biopsy specimens permits precise localisation of tissue antigen-antibody complexes and quicker results for diagnosis. This method, with some modifications, may be useful for immunofluorescence study of other tissues such as skin and lymph node biopsy specimens.

We thank $\mathrm{Mr} \mathrm{CH}$ Ning for photographic assistance and Mrs Clara Chiu for preparing the manuscript.

\section{References}

1 McLuskey RT. The value of immunofluorescence in the study of human renal disease. $J$ Exp Med 1971;134:242S-56S.

$2 \mathrm{Ng}$ WL, Chan CW, Yeung CK, Hua SP. The pathology of primary IgA glomerulonephritis-a renal biopsy study. Pathology 1981:13:137-43. 
3 Davies DR, Tighe JR, Wing AJ, Jones NF. Immunoglobulin deposition in membranous glomerulonephritis: immunofluorescence and immunoelectron microscopy findings. Histopathology 1977;1:39-52.

4 Harrison DJ, Thomson D, Macdonald MK. Membranous glomerulonephritis. J Clin Pathol 1986;39:167-71.

5 Dunnill MS. Pathological basis of renal disease. 2nd ed. London: Baillière Tindall, 1984:555-6.

6 Mayers CP. Histological fixation by microwave heating. $J$ Clin Pathol 1970;23:273-5.

7 Login GR, Dvorak M. Microwave energy fixation for electron microscopy. Am J Pathol 1985;120:230-43.

8 Leong ASY, Daymon ME, Milios J. Microwave irradiation as a form of fixation for light and electron microscopy. $J$ Pathol 1985;146:313-21.

9 Hafiz S, Spencer RC, Lee M, Gooch H, Duerden B. Use of microwaves for acid and alcohol fast staining. J Clin Pathol 1985;38:1073-6.
10 Estrada JC, Brinn NT, Bossen EH. A rapid method of staining ultrathin sections for surgical pathology TEM with the use of the microwave oven. Am J Clin Pathol 1985;83:639-41.

11 Boon ME, Kok LP, Ouwerkerk-Noordam E. Microwavestimulated diffusion for fast processing of tissue: reduced dehydrating, clearing and impregnating times. Histopathology 1986;10:303-9.

12 Leong ASY, Milios J. Rapid immunoperoxidase staining of lymphocyte antigens using microwave irradiation. $J$ Pathol 1986;148:183-7.

Requests for reprints to: Dr KY Chiu, Histopathology Unit, Department of Pathology, University of Hong Kong, Queen Mary Hospital Compound, Pokfulam Road, Hong Kong.

\section{Computer programs for accurate determination of size of DNA fragments in agarose gels}

\begin{abstract}
JULIA LING, ${ }^{*} \quad \mathrm{~K} \quad$ L $\quad$ E LING, $\dagger$ K $\quad$ W CHAN, $\ddagger \quad G \quad$ L FRENCH* From the *Department of Microbiology, The Chinese University of Hong Kong, Prince of Wales Hospital, the †Department of Medicine, University College and Middlesex School of Medicine, The Rayne Institute, London, and the $\ddagger$ Department of Pathology, University of Hong Kong, Queen Mary Hospital, Hong Kong
\end{abstract}

The use of plasmid profiling for epidemiological studies of bacterial infections is an accurate and reliable method of typing, especially where no other typing scheme is available. ${ }^{1}$ Restriction endonuclease digestion of whole DNA has also been used in fingerprinting strains of the same bacterial species. ${ }^{2} 3$ All these studies require accurate estimation of the size of DNA fragments. Usually, molecular size of these fragments is determined by subjecting the DNA samples to electrophoresis on agarose, or polyacrylamide gels. The molecular size of DNA is inversely proportional to the distance travelled and may be calculated by comparison with the migration of known standards on the same gel. ${ }^{4}$ The logarithm of size in megadaltons is plotted against the logarithm of relative mobility for each of the standards. The size of the unknown DNA fragments, which is on the same gel as the standards, is read off from the curve of best fit. Such a standard curve is usually concave upward, and only a portion of it is roughly linear. The relative mobility of DNA fragments is calculated by

Accepted for publication 27 November 1986 following formula:

$$
\frac{10}{\mathrm{X}} \times \mathrm{Y}
$$

where $\mathrm{X}=$ distance travelled by one of the standards

$$
\begin{aligned}
& \mathrm{Y}= \text { distance travelled by the DNA fragment } \\
& \text { of which the relative mobility is to be } \\
& \text { calculated }
\end{aligned}
$$

This method is very inaccurate, however, if the curve of best fit is drawn subjectively. More accurate methods have been devised that relate migration and molecular size on the basis of standard mathematical formulae. These complex equations usually require computer analysis for their solution. ${ }^{5}$ Such programs are available in BASIC ${ }^{6}$ and FORTRAN. ${ }^{5}$

We have written two computer programs in MBASIC that will run on Apple II/II + /IIe computers, and these may be easily adapted for other microcomputers. The programs are relatively short and produce two solutions, one fitting a straight line and the second a parabola to results from a set of standards (figs 1 and 2). They are written based on the following equations:

$$
\begin{aligned}
y=m x & +c \text { where } \\
y=\text { molecular weight of DNA } & >\text { for linear } \\
x=\text { relative mobility of DNA } & >\text { relation } \\
m \text { and } c=\text { constants } & \\
y=a+b x+c x^{2} \text { where } & >\text { for } \\
y=\text { molecular weight of DNA } & >\text { parabolic } \\
x=\text { relative mobility of DNA } & >\text { relation }
\end{aligned}
$$

The migration distance of each standard from individual gels and its size are entered into the program. The migration of one standard is chosen and entered as "STANDARD MIGRATION" when prompted. The relative mobility will be calculated by the program. Once data are entered, results are produced almost instantaneously. 